\title{
Investigation of faulty behavior of the sensorless control switched reluctance motor drives
}

\author{
Alexander Krasovsky \\ Department of Electrical Engineering and Power Electronics Bauman Moscow State Technical University, Moscow, \\ Russian Federation
}

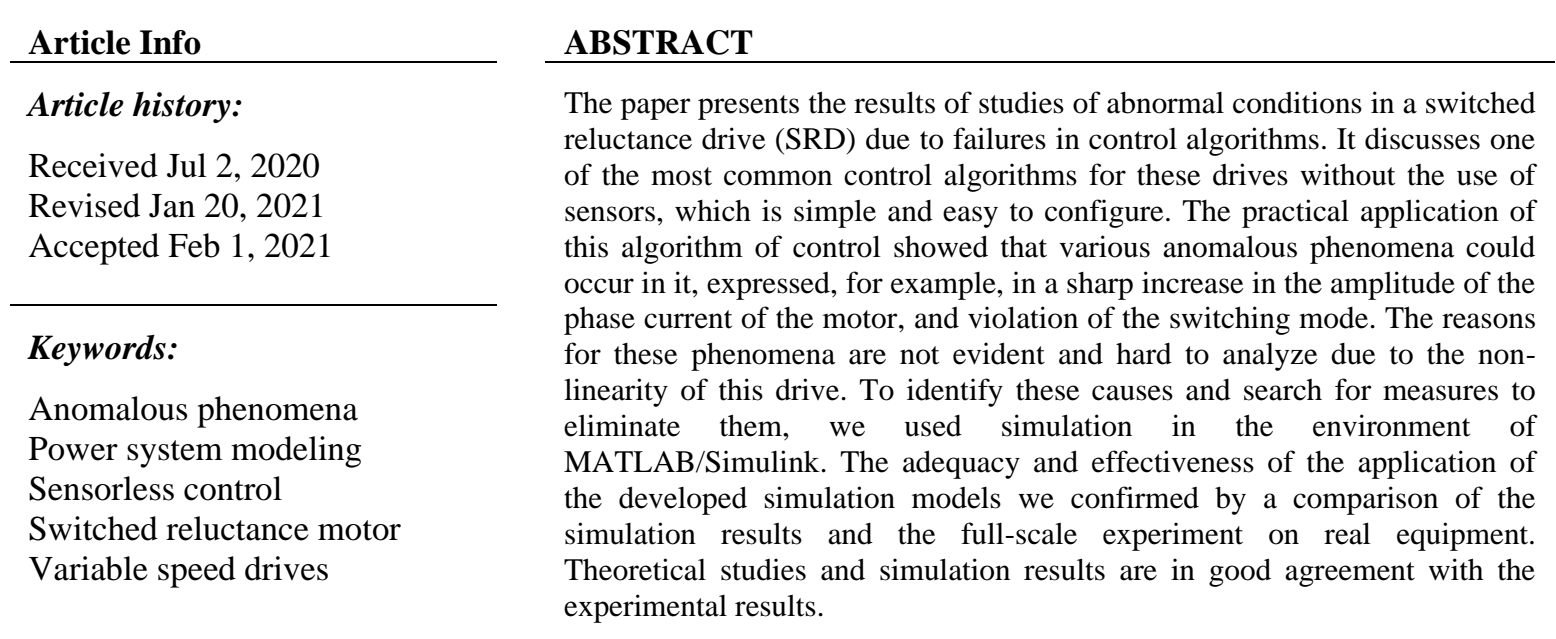

This is an open access article under the CC BY-SA license.

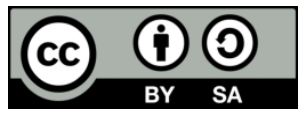

\section{Corresponding Author:}

\author{
Alexander Krasovsky \\ Department of Electrical Engineering and Power Electronics \\ Bauman Moscow State Technical University, Moscow, Russian Federation \\ Email: krasovsky@bmstu.ru
}

\section{INTRODUCTION}

Among the problems solved during the design of the electric drive, one of the most important is the determination of the permissible deviations of the values of its parameters and control signals from their calculated values, at which it maintains its working capacity. By working capacity, we understand the ability of an electric drive to implement the required laws of change in its adjustable coordinates (torque and speed) with the maximum permissible deviations established in the technical documentation for its design. All other modes of its operation are abnormal, which, in addition to the violation serviced by the electric drive of the technological process, in the most severe cases can lead to emergencies. For traditional electric drive systems, a lot of literature is devoted to these issues, for example [1]-[4] and others. The switched reluctance drive (SRD), which has been rapidly developing in recent years [5]-[8], has a number of specific features, therefore, the issues of eliminating the abnormal modes of its operation require special study. Among the possible reasons for the abnormal operating modes of the SRD within the framework of this paper, we restrict ourselves only to the reasons associated with failures in control algorithms.

The most specific element of these drives is a switched reluctance motor (SRM). An important advantage of this motor is its high reliability, which is due to its extremely simple design. An SRM has concentrated phase windings on stator teeth and a gear passive ferromagnetic rotor [9]-[11]. The operation of the SRM consists of alternately connecting its phase windings by means of an inverter to the voltage source 
in strictly defined angular positions of the rotor relative to the stator. For this reason, in a typical version of $\mathrm{SRD}$, we use a special rotor position sensor (PS) to determine the position of the rotor at the switching points of the phase windings [9]. However, we know that the presence of a real PS in the structure of the electric drive reduces its reliability, increases weight, dimensions, and costs [12], [13]. To date, various authors have developed quite a few methods for indirectly determining the position of the rotor in order to exclude PS from the SRD control structure [14]-[17]. Well-known sensorless control methods use parameter measurements of various signals in the excited or unexcited phases of SRM. In the first case, real curves of phase currents and voltages or reconstructed signals are usually used in a mathematical model of a drive operating in real-time in parallel with a real system [14], [15]. In the second case, the reaction of the unexcited phase to various types of test signals is analyzed in [16].

The main disadvantage of such a control is the difficulty of its implementation at high speed when the current in the functioning phase lasts almost the entire period, and there is no time for test pulses. In their paper, M. Ehsani and B. Fahimi [16] examined in detail the advantages and disadvantages of these and other control methods, as well as the trends for their further improvement. One of the most popular and widely known approaches of sensorless control of SRM is the method, which is known in the literature as the flux linkage/current method [9], [17]-[19]. In it, the phase switching position $\Theta_{\text {com }}$ corresponds to the moment equality calculated value of flux linkage of the phase $\Psi_{\text {calc }}$ and its required value in the phase of switching

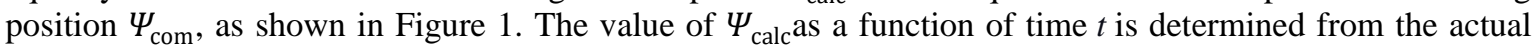
values of the phase current $I_{\mathrm{ph}}(t)$, phase voltage $U_{\mathrm{ph}}(t)$, and voltage drops across active phase resistance $R_{\mathrm{ph}}$

$$
\Psi_{\text {calc }}(t)=\int\left[U_{\mathrm{ph}}(t)-I_{\mathrm{ph}}(t) R_{\mathrm{ph}}\right] d t
$$

In the general case, the family of characteristics $\Psi_{\text {com }}$ for different values of the phase current $I_{\mathrm{ph}}(t)$ and the switching positions $\Theta_{\text {com }}$, usually called switching lines, pre-determined one way or another and stored as an array of numbers in the corresponding storage device of control system SRD. In practice, such control is generally realized by software. It is important to emphasize here that in this method of determining the phase switching positions of the SRM, as in any other sensorless control variant, these are determined indirectly by special processing of electrical signals that depend on the rotor position. Therefore, the identity of the sensor control and sensorless control is achievable only if well-defined additional conditions and restrictions are met. Hence, when these restrictions are exceeded, the working capacity of the electric drive is violated.

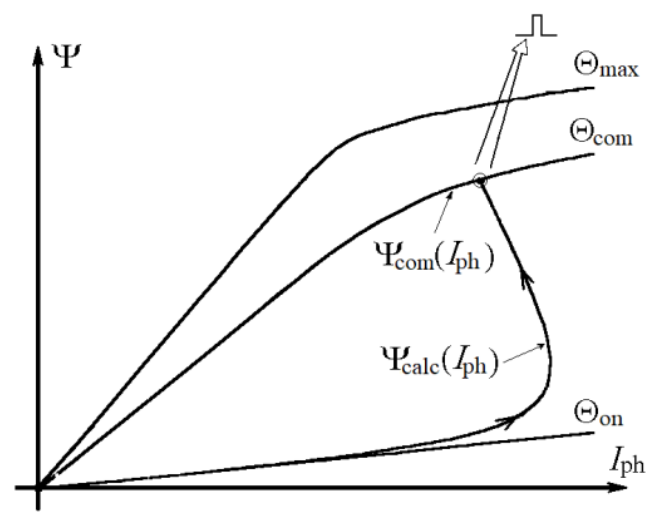

Figure 1. Determination of the switching position of the SRM $\left(\Theta_{\text {on }}-\right.$ switching - on position, $\Theta_{\text {com }}{ }^{-}$ switching positions, $\Theta_{\max }-$ full aligned position stator and rotor teeth)

The author of this paper has set himself the goal of showing with a specific example that even when using a version of the SRD sensorless control that is simple from the point of view of physical interpretation, various non-obvious anomalous phenomena, and drive malfunctions can occur. To do this, the author conducted a detailed analysis of the features of the control algorithm under consideration and identified the most characteristic causes that could lead to its failures. Given the specifics of SRD, a tool for research in the form of simulation models is justified, a brief description of the given experimental equipment. After that, the research results and their analysis are presented, which allow us to identify the physical causes of the occurrence of abnormal phenomena. Based on this, relevant recommendations and conclusions are drawn. 


\section{FEATURES OF CONSIDERED SENSORLESS CONTROL SRD}

Figure 2 presents the functional scheme of the SRD block generating a signal of the position of the rotor with the considered algorithm of sensorless control. In it, the comparator $\mathrm{K}$ determines the switching position when the signals $\Psi_{\text {calc }}\left(I_{\mathrm{ph}}, \Theta_{\text {com }}\right)$ and $\Psi_{\text {com }}\left(I_{\mathrm{ph}}, \Theta_{\text {com }}\right)$ are equal. The flux linkage $\Psi_{\text {calc }}$ is calculated based on the real values of $I_{\mathrm{ph}}(t)$ and $U_{\mathrm{ph}}(t)$, as well as the values of the scaling coefficients of the algorithm: by voltage $K_{U}$, current $K_{I}$, and phase resistance $K_{R}$

$$
\Psi_{\text {calc }}(t)=\int\left[K_{U} U_{\mathrm{ph}}(t)-K_{I} K_{R} I_{\mathrm{ph}}(t) R_{\mathrm{ph}}\right] d t
$$

When using a direct sensor induced in the phase of the EMF, or with full $I_{\mathrm{ph}} R_{\mathrm{ph}}$ compensation, the current value of $\Psi_{\text {calc }}$ depends on only one coefficient by EMF - $K_{E}$ :

$$
\Psi_{\text {calc }}=\int K_{E} E d t
$$

Where $E=\left(U_{\mathrm{ph}}-I_{\mathrm{ph}} R_{\mathrm{ph}}\right)$ is the EMF phase, the correct operation of the algorithm involves the use of a curve $\Psi_{\mathrm{com}}\left(I_{\mathrm{ph}}, \Theta_{\mathrm{com}}\right)$, identical to the real dependence, as well as the representation of the curves $\Psi_{\text {calc }}\left(I_{\mathrm{ph}}, \Theta_{\text {com }}\right)$ and $\Psi_{\text {com }}\left(I_{\mathrm{ph}}, \Theta_{\text {com }}\right)$ on one scale. In the general case, the adjustable parameters of the control system when setting of the SRD phases switching algorithm are the coefficients $K_{U}, K_{I}, K_{R}$ (either the only coefficient $\left.K_{E}\right)$, as well as the shape of the switching line $\Psi_{\text {com }}\left(I_{\mathrm{ph}}, \Theta_{\text {com }}\right)$. Without touching upon the specifics of choosing specific values of $K_{U}, K_{I}, K_{R}$ coefficients and their connection with parameters of the SRM control part, we only note that they are, in essence, scale factors that determine the proportion of the corresponding physical quantities in the calculation procedure determining the values of $\Psi_{\text {calc }}$ and $\Psi_{\text {com }}$. By changing the values of these coefficients, it is possible to change the shape and scale of representation of $\Psi_{\text {calc }}$ and $\Psi_{\text {com }}$.

Certainly, with the same parameters of the SRM phases, correctly determined values of the coefficients $K_{U}, K_{I}, K_{R}$ (or only the value of the coefficient $K_{E}$ ) and the precisely specified shape of the Switching line $\Psi_{\text {com }}\left(I_{\mathrm{ph}}, \Theta_{\text {com }}\right)$, the conditions for switching the SRM phases in the sensorless control variant do not differ from the sensor version for the same source data. Miller T. [9] presented recommendations on tuning this sensorless control algorithm. Violation of the above conditions leads to deviation of the parameters of the switching of the SRM from the set. Therefore, it is very important to establish acceptable limits for changes in each of these parameters of the algorithm that do not violate the normal operation of the SRD.

We should note another important feature of the practical implementation of this control method. To avoid false alarms of the algorithm for small values of the phase currents in the switching line, a "dead zone" or limitation of $\Psi_{\text {com }}$ at a certain minimum level $\Psi_{\min }$, was introduced, as shown in Figure 3 . In the region of small values of phase current $I_{\mathrm{ph}}$ flux linkage is constant, i.e. $\Psi_{\mathrm{com}}=\Psi_{\min }=$ const. The correct choice of the value of $\Psi_{\min }$ is of fundamental importance for the considered SRD control algorithm. As can you see, in the control algorithm under consideration there are a lot of variable parameters, on the correct choice of which its working capacity depends, and in case of errors, the probability of occurrence of failures and false positives is high [18]. However, the specific causes and mechanisms of development of these processes remain fully unexplored and not covered in the literature. To identify the causes of such phenomena, it is necessary to conduct a detailed analysis of the features of the work, the practical settings of this algorithm and to develop universal tools for its study with the maximum approximation to real conditions.

Given the non-linearity of SRM, the presence of continuous and discrete signals, the mathematical description of the operation of the sensorless SRD control system under consideration is rather cumbersome, uninformative, and inconvenient for such studies [9], [10]. Therefore, when debugging and tuning this algorithm, it is highly desirable to have a simulation model of the drive. In order to reflect the real features of the considered part of the SRD control system in the model, it is advisable to make the structure of the model as close as possible to the structure of a real device with imitation of the logic and time sequence of work of its elements. The most convenient tool for this is the MATLAB mathematical package with the SIMULINK environment [20], [21]. 


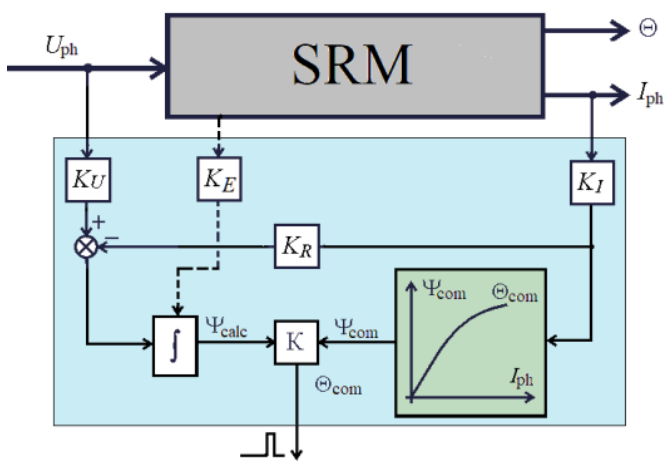

Figure 2. Functional scheme of the rotor position signal-generating block with sensorless control of SRM

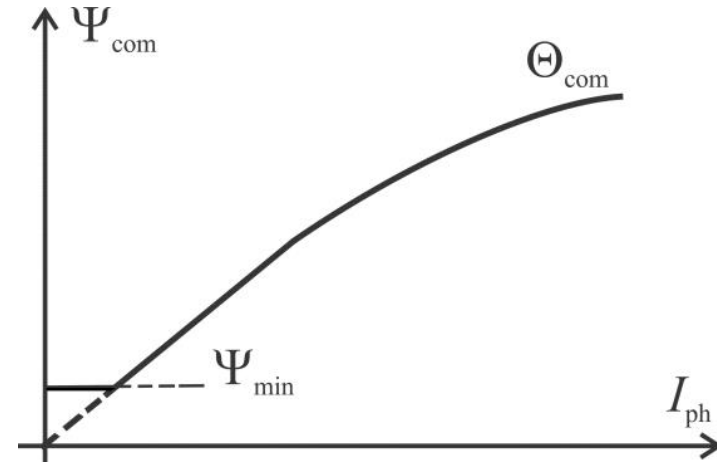

Figure 3. Real shape of switching line

\section{DESCRIPTION OF THE SRD SIMULATION MODEL}

The general principles for constructing the SRD simulation model are considered in a fairly large number of works, in particular, their description can be found in [22]-[25] and others. The main difference between the model created in the process of performing this work and the known ones is in the design of the SRM phase voltage generating block. A full model of the voltage-on-phase generation block with sensorless SRD control is shown in Figure 4. The inputs of the block receive signals: about switching position of this phase (port In 1); about switching the previous phase (block From 1); about the instantaneous value of the phase current (block From 2); about voltage power supply (block From 3).

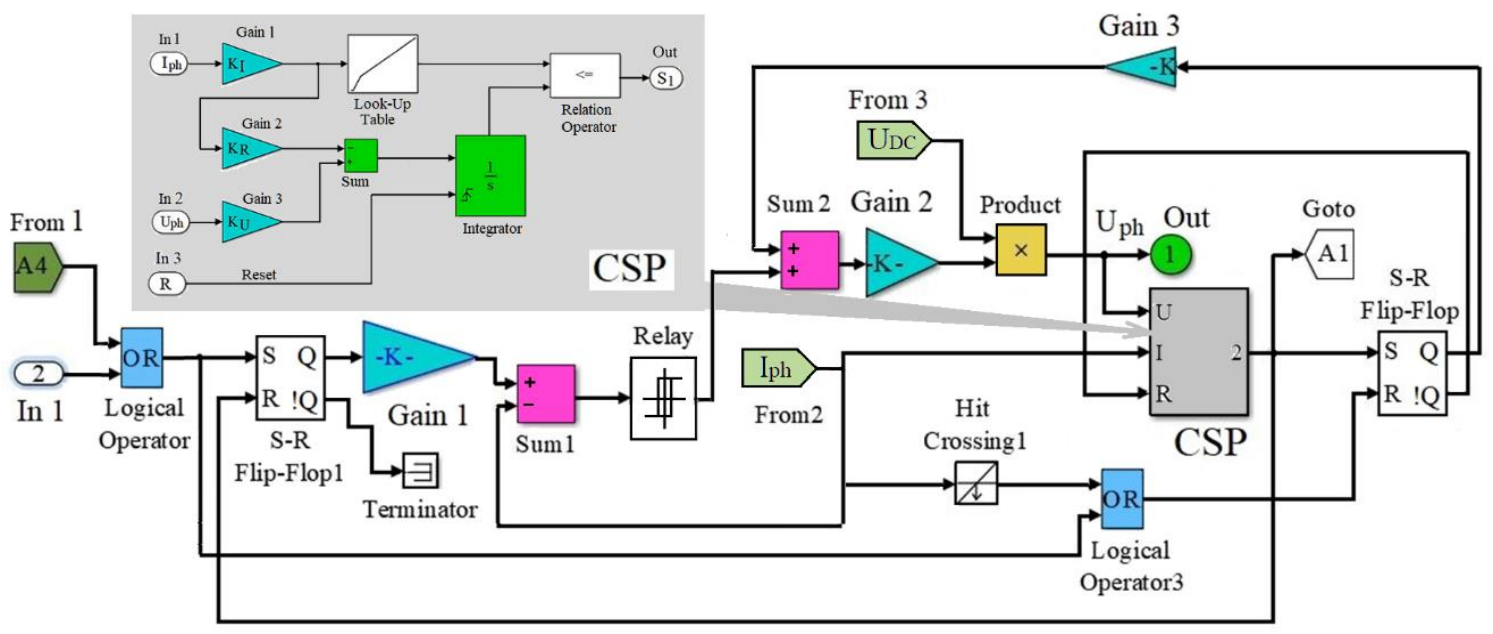

Figure 4. Simulation model of the phase voltage-generating block

The phase current limit is set by the block Gain 1. Blocks Gain 1, Sum 1, and Relay provide an imitation of the current-limiting mode in the motor phase [9]. The phase turns on and turns off, respectively, when the triggers Flip-Flop 1 and Flip-Flop 2 are triggered. The remaining blocks included in the model, perform auxiliary functions. To simulate the sensorless control mode, this model provides for a special subsystem for calculating the switching position CSP, which reproduces the mechanism described above for indirect determination of the position of the switching of the phase SRM. The upper part of Figure 4 shows a variant of building a subsystem CSP. The logic of its operation repeats the operation of a real device, the functional diagram of which shown in Figure 2. This block receives phase voltage, current, and pulse signals to reset the integrator, which is part of it. The switching line $\Psi_{\text {com }}\left(I_{\mathrm{ph}}, \Theta_{\mathrm{com}}\right)$ is set in the form of the corresponding table of the Look-Up Table element settings. 
The integrator performs the calculation of the current value of the flux linkage of the phase $\Psi_{\text {calc }}\left(I_{\mathrm{ph}}, \Theta_{\text {com }}\right)$ according to expressions (2) or (3). The values of the tuning coefficients of the algorithm $K_{\mathrm{U}}$, $K_{I}, K_{\mathrm{R}}$ are set by the transmission coefficients of the elements Gain 1, Gain 2, and Gain 3. The phase switching signal is generated by the Relational Operator block and is transmitted to the output of Out 1 at the time of equality $\Psi_{\mathrm{com}}\left(I_{\mathrm{ph}}, \Theta_{\mathrm{com}}\right)$ and $\Psi_{\text {calc }}\left(I_{\mathrm{ph}}, \Theta_{\mathrm{com}}\right)$. To eliminate the accumulation of errors in position determination as well as in a real device, it is necessary to reset the integrator at the end of each phase switching cycle. The trigger Flip-Flop 2 generates the reset signal at the time of switching the block Relational Operator.

To ensure a uniform scale of change of parameters and variables in simulation models of SRD, as well as to give generality to the simulation results, we used the transition to relative values. The basic value of the magnetic conductivity of phase is equal to its value in the coordinated position of the teeth of stator and rotor, i.e., $\lambda_{\text {base }}=\lambda_{\text {max }}$. The basic values of the angular intervals of movement of the rotor, except of interval of switching on phase $\gamma_{\text {on }}$, equal to the angular interval of partial overlap of the interacting teeth of the stator and rotor, i.e., $\gamma_{\text {base }}=\gamma_{\text {overlap }}$. The base value of the interval of switching on the phase $\gamma_{\text {on base }}$, is equal to the angular interval between the angle of the beginning of overlapping of the teeth $\Theta_{\text {unal }}$ and the angle $\Theta_{0}$ where $\Theta_{0}$ is the angle of intersection of the continuation of the growing section of the dependence $\lambda(\Theta)$ with the $\Theta$-axis, i.e. at $\lambda(\Theta)=0$. The base voltage value is equal to the rated voltage of the motor $U_{\text {base }}=U_{\text {rated }}$. The base values of current, torque, and speed are taken as their values at the point of transition of the magnetic system of the SRM from linear mode to local saturation of interacting teeth $I_{\text {base }}=I_{\text {sat }}$, where $I_{\text {sat }}$ is the current at which is begins local saturation of teeth. Thus, the base value of the interval of switching on the phase $\gamma_{\text {on base }}$, ensures the constancy of the phase current with the amplitude $I_{\text {sat }}$ in the overlapping zone of the stator and rotor teeth at the rated voltage $U_{\text {rated }}$ and the corresponding speed value $\omega_{\text {rated. }}$ All relative values of parameters and variables are subsequently marked with an asterisk $(*)$.

\section{EXPERIMENTAL EQUIPMENT FOR VERIFICATION OF SIMULATION RESULTS}

To verify the simulation results and conclusions made on their basis, we used experimental SRD samples. These samples are an integral part of a computerized test complex for experimental studies of SRD with a capacity of up to $15 \mathrm{~kW}$. The computer-aided test complex allows to measure up to 16 analog signals; automatically install and maintain a given load; automatically register fast and slow dynamic processes, choose the values necessary for recording, save the results of experiments in various data formats. Inverter with power modules BSM75GB1201 has the following parameters: power up to $15 \mathrm{~kW}$, supply voltage - 380 $\mathrm{V}$, frequency - $50 \mathrm{~Hz}$, power - up to $15 \mathrm{~kW}$, rated current - 50A. Universal microcontroller Intel 8XC196MH. The experimental four-phase SRM with a rated power of $5 \mathrm{~kW}$ has the following main constructive parameters: the number of stator poles 8 , the number of rotor poles 6 , the outer diameter of the active part of the stator is $206 \mathrm{~mm}$, the outer diameter of the rotor is $116 \mathrm{~mm}$, and the air gap is $0.4 \mathrm{~mm}$.

To obtain current signals used in the SRD control algorithm and during test registration, the test complex is provided with current sensors included in each phase of the converter, in the DC link and the capacitive filter circuit of the converter. Current sensors are based on LEM type LT100-P Hall sensors. The signals from the sensors enter the current/voltage conversion board, and then to the analog inputs of the microcontroller and into a computerized measurement recording system. There, a signal is transmitted proportional to the voltage in the DC link, obtained employing a voltage sensor.

\section{RESULTS AND DISCUSSION}

We conducted the main studies on SRD simulation models, and then we compared some modeling results with experimental results. Given a sufficiently large variety of possible combinations of deviations of the parameters of the considered algorithm from the calculated values in this article, at the first stage, we consider the influence of only deviations the tuning coefficients of the sensorless control algorithm. Moreover, due to the wide variety of possible combinations of the coefficients $K_{U}, K_{I}, K_{R}$ we will consider for a simpler case - we will consider only deviations of the coefficient $K_{E}$, which corresponds to the use of the signal directly by EMF under the expression (3).

Besides, for definiteness, we conditionally assumed that the identity of the SRM control model in the presence of a position sensor and a mode without a sensor occurs at a coefficient $K_{E}=1$. Then, by changing the values of $K_{E}$ in both directions relative to this value, we can evaluate its effect on the conditions of switching SRM. The error in the calculation $\Psi_{\text {calc }}$ by the signal of the EMF sensor may be due to errors in the corresponding sensors and intermediate converters at the input of the control microprocessor system. As follows from (3), the value of the coefficient $K_{E}$ affects the rate of increase in time of the $\Psi_{\text {calc }}$ value, which 
accordingly affects the position of the phase switching $\Theta_{\text {com. }}$. As an example, in Figure 5(a) with the solid lines shown the curves $\Psi_{\text {calc }}^{*}\left(\Theta^{*}\right)$ and $\Psi_{\text {com }}^{*}\left(\Theta^{*}\right)$ are obtained by modeling processes in the SRM at $K_{E}=1$ and $\omega^{*}=2$. In this case, the position $\Theta^{*}$ com is determined without error.

From relation (2) it follows that with an increase in the value of $K_{E}\left(K_{E}>1\right)$, the rate of increase of $\Psi_{\text {calc }}^{*}\left(\Theta^{*}\right)$ increases. The equality between $\Psi_{\text {calc }}^{*}\left(\Theta^{*}\right)$ and $\Psi_{\text {com }}^{*}\left(\Theta^{*}\right)$ comes earlier, as a result of which the working phase of the SRM and the subsequent phase are switched on with advance. An earlier inclusion of the phase leads to an increase in the current in it and, accordingly, with the same shape of the switching line, it increases the flux linkage $\Psi_{\text {com. }}^{*}$. As a result, a certain new stable state of the mode of switching of the SRM occurs $\Psi_{\text {calc }}^{*}=\Psi_{\text {com }}^{*}$ when the position $\Theta^{*}$ com is shifted by a certain phase angle $\Delta \gamma_{\text {adv }}^{*}$ in the direction of advance. This is confirmed by the flux linkage curves $\Psi_{\text {calc }}^{*}\left(\Theta^{*}\right)$ and $\Psi_{\text {com }}^{*}\left(\Theta^{*}\right)$ at $K_{E}=1,2$, shown in dashed lines in Figure 5 (a). We found that an overestimation of the $K_{E}$ value by $20 \%$ led to an approximately $60 \%$ increase in amplitude of the phase current $I_{\mathrm{ph}}^{*}\left(\Theta^{*}\right)$.

On the contrary, it can also be seen from relation (2) that, with a decrease in the value of $K_{E}$, the rate of increase of $\Psi_{\text {calc }}^{*}\left(\Theta^{*}\right)$ decreases. The equality between $\Psi_{\text {calc }}^{*}\left(\Theta^{*}\right)$ and $\Psi_{\text {com }}^{*}\left(\Theta^{*}\right)$ comes with a delay and the working phase is switched off and the subsequent phase is turned on and also lags concerning the position of $\Theta^{*}$ com by the phase angle $\Delta \gamma^{*}{ }_{\text {lag }}$. Figure $5(\mathrm{~b})$ by dashed lines shows the curves $\Psi_{\text {calc }}^{*}\left(\Theta^{*}\right)$ and $\Psi_{\text {com }}^{*}\left(\Theta^{*}\right)$ corresponding to this switching mode when $K_{E}=0,8$. In the same place, for comparison, by analogy with Figure 5 (a), solid lines show similar curves at $K_{E}=1$. In this case, the decrease in the amplitude of the phase current $I_{\mathrm{ph}}^{*}\left(\Theta^{*}\right)$ is about $20 \%$.

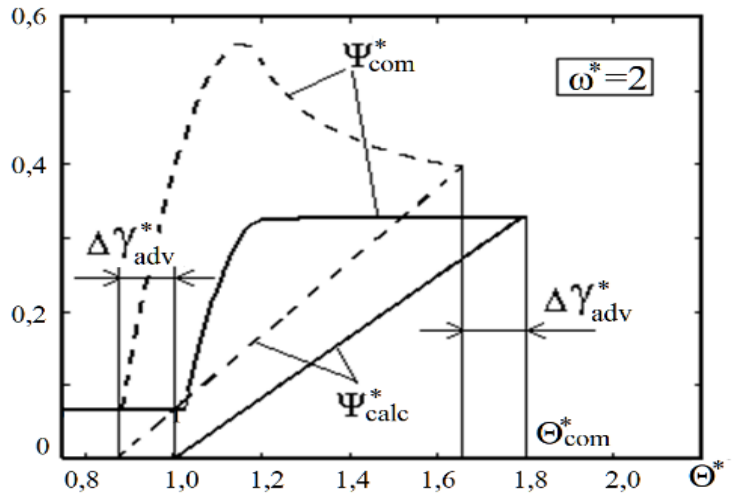

(a)

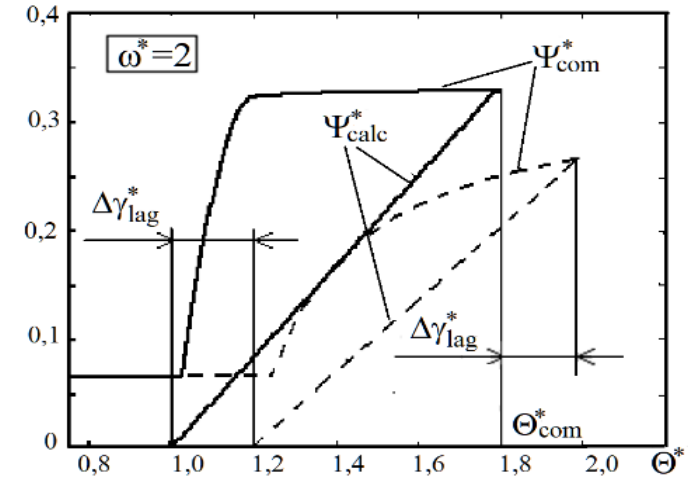

(b)

Figure 5. Displacement of the switching position $\Theta^{*}$ com at (a) increasing $K_{E}$ and (b) decreasing $K_{E}$

A series of additional experiments on the SRD simulation model made it possible to determine the boundary values of the coefficient $K_{E}$ and to clarify the behavior of the drive when going beyond these boundaries. As an example, Figure 6 shows the family of mechanical characteristics in the form of a dependence of the average value of the torque on speed $T_{\mathrm{av}}^{*}\left(\omega^{*}\right)$ for different values of the $K_{E}$ coefficient for a view with a configuration of $8 / 6$ with typical parameters. These characteristics reflect the basic properties of the SRD when the $K_{E}$ changes.

Analysis Figure 6 allows us to conclude that the SRD working area for any characteristic $T_{\mathrm{av}}^{*}\left(\omega^{*}\right)$ (at any value of $K_{E}$ ) in the zone of relatively high speeds is limited by a certain maximum value of speed corresponding to this characteristic, which increases with increasing of value $K_{E}$. When $K_{E}<1$ and $K_{E}>1$, the working area of the drive has a limitation in the zone of low speeds. When we go beyond the working area of each of the characteristics, abnormal SRD operation modes occur, which are manifested either in a failure of the switching mode or in a sharp surge of the phase current of the SRM. Next, we consider the causes of the above limitations of the working area of the SRD on its mechanical characteristics in the field of high and low speeds and analyze the possible development of an abnormal situation in the drive when leaving the working area. Figure 7 (a) shows an enlarged scale of the high-speed zone of mechanical characteristics shown in Figure 6. You can see that for any of the characteristics presented, the minimum value of the torque does not remain constant, but changes relatively little. The simulation made it possible to establish that the reason for the limitations of the SRD working area in the high-speed region is the limitation of the switching line $\Psi_{\text {com }}\left(I_{\mathrm{ph}}, \Theta_{\text {com }}\right)$ at the level of $\Psi_{\text {com }}=\Psi_{\text {min }}=$ const as shown in Figure 3. As soon as the amplitude of the phase current, decreasing with increasing speed, reaches a value at which its 
corresponding value $\Psi^{*}$ com om becomes equal to $\Psi^{*}{ }_{\min }$ as shown in Figure 8 , the position of $\Theta^{*}$ com is determined with an error in the direction of the lag of the switching position of each phase by about the switching position of the previous phase. As a result, the phase angle between two adjacent phases switching $\gamma_{\text {com }}^{*}$ increases with each new switching cycle $\gamma_{\text {com,1 }}^{*}<\gamma_{\text {com, } 2}^{*}, \ldots$.

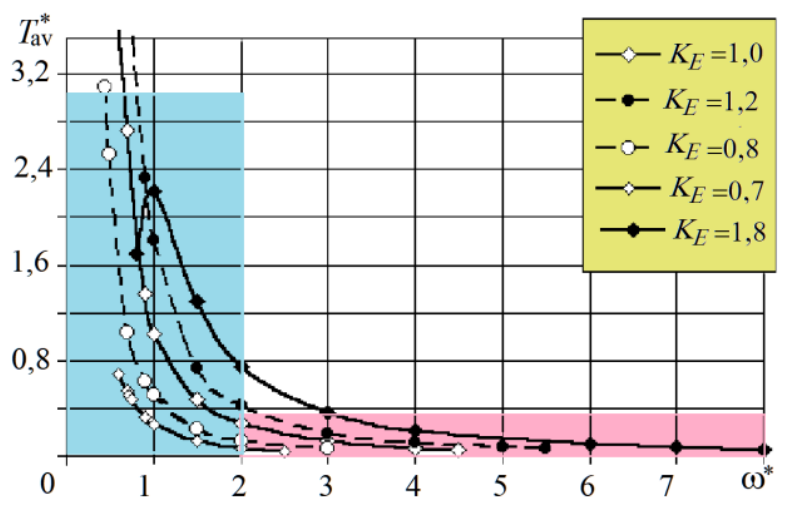

Figure 6. The mechanical characteristics $T_{\mathrm{av}}^{*}\left(\omega^{*}\right)$ of the SRD at changing the value of coefficient $K_{E}$

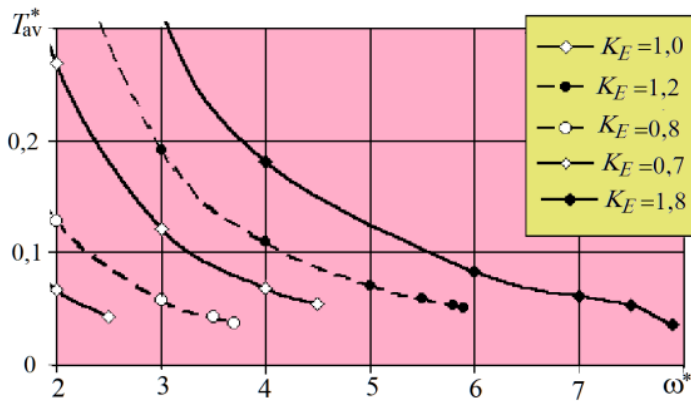

(a)

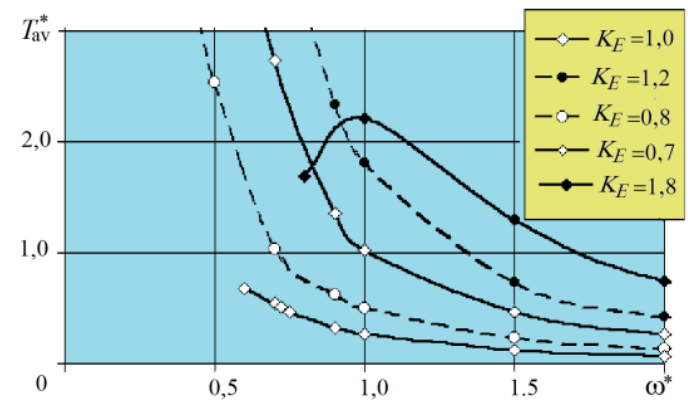

(b)

Figure 7. Fragments of mechanical characteristics $T_{\mathrm{av}}^{*}\left(\omega^{*}\right)$ of the SRD in the zone of high (a) and low (b) speeds when changing the value of coefficient $K_{E}$

The angular displacement of the switching position of the phase windings of SRM in the direction of delay with each new switching cycle also increases. As a result, the position of the phase switching is gradually shifted towards the generator mode of operation of the SRM. In the first of the phases, the switching cycle of which (falls into the generator mode, we observe a sharp surge of current, as shown in Figure 9 (a). The experimental curves of the phase currents of the SRM, shown in Figure 9 (b), confirm the above-described mechanism for the development of the anomalous situation in the drive in the region of high speeds. As can be seen, the nature of the change in phase currents obtained by modeling as shown in Figure 9 (a) and the experimental dependence as shown in Figure 9 (b) are quite similar in appearance. To exclude such an abnormal mode of operation of the SRD, an appropriate choice of the value $\Psi^{*}{ }_{\text {min }}$. Throughout the entire operating range of shaft loads of SRM, the algorithm of sensorless switching of the SRM should not fall into the "dead zone" of the switching line. The most reliable upper estimate of $\Psi^{*}{ }_{\text {min }}$, can be such a value that ensures stable switching of the view in real idle mode of the drive (the load is caused only by the total losses in the drive).

The low-speed zone of characteristic $T_{\mathrm{av}}^{*}\left(\omega^{*}\right)$ on an enlarged scale is shown in Figure 7 (b). You can see that at $K_{E}$ values sufficiently close to unity, the mode of stable switching of the SRM is observed almost to zero speed. However, as the deviations of $K_{E}$ values from unity increase in both directions, when the speed reaches a certain minimum value, switching failure occurs. Moreover, the mechanisms of development of abnormal situations in - SRD and their manifestation at $K_{E}>1$ and at $K_{E}<1$ are different. At $K_{E}<1$, the 
position of switching the phase of the SRM phase shifts toward the delay with respect to the position at $K_{E}=$ 1. If the phase does not have time to turn off before the SRM enters the generator mode, this causes a sharp increase in the current in it and a switching failure (emergency mode SRD). The mechanism of transition of the SRD into emergency mode at $K_{E}=0,7$ shown in Figure 10 (a) and Figure 10 (b).

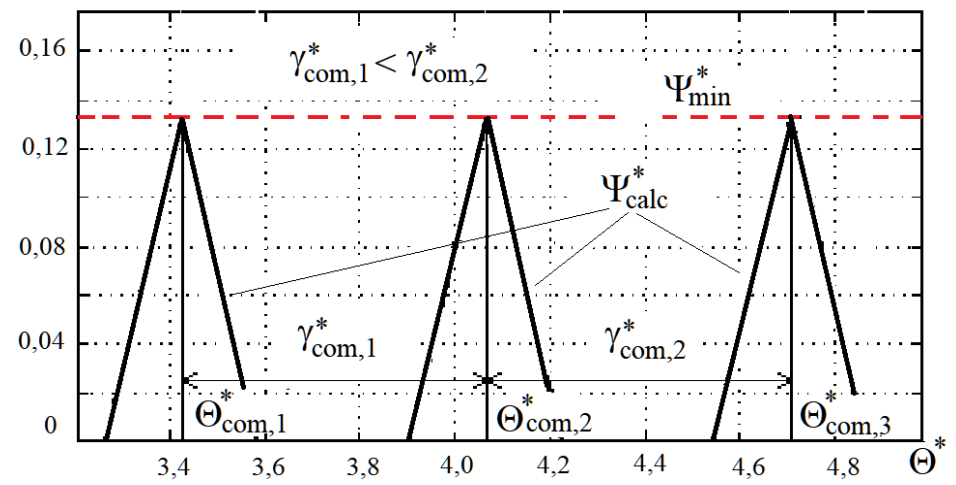

Figure 8. Determination of switching position $\Theta^{*}$ com at low values $\mathrm{Y}_{\mathrm{com}}$.

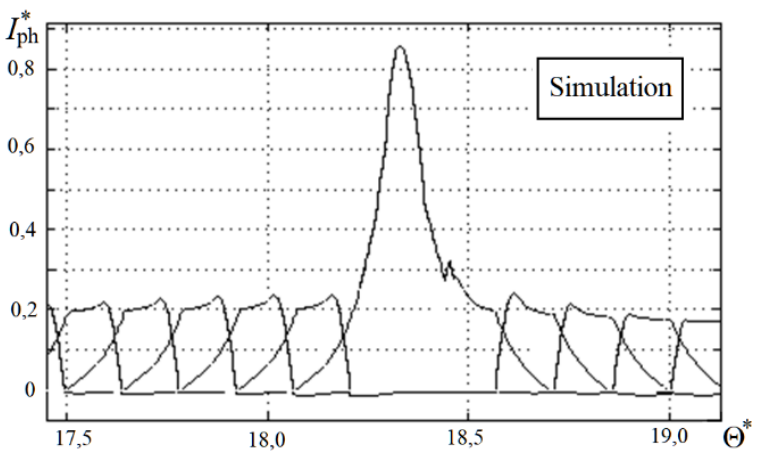

(a)

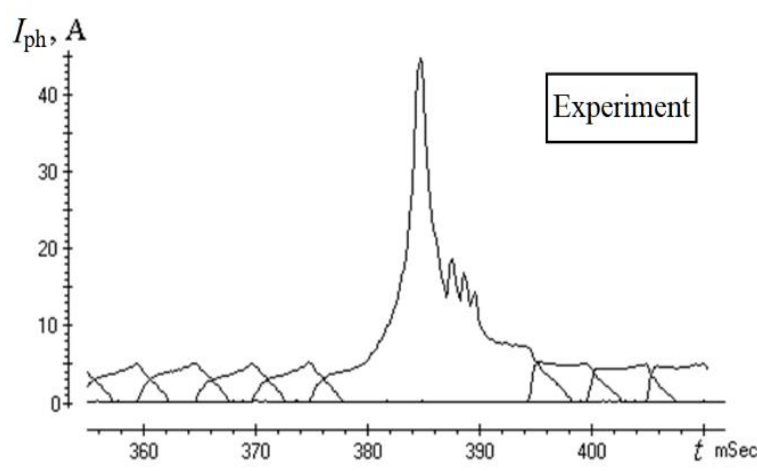

(b)

Figure 9. Anomalous SRD mode in the high-speed zone (a) simulation, (b) experiment

The solid lines in the figures show, respectively, the phase current curves in Figure 10 (a), as well as the dependencies $\Psi_{\text {com }}^{*}\left(\Theta^{*}\right)$ and $\Psi_{\text {calc }}^{*}\left(\Theta^{*}\right)$ in Figure $10(\mathrm{~b})$, at the boundary of the emergency mode at $\omega^{*}=0,6$. As can you see, the equality between $\Psi_{\text {com }}^{*}\left(\Theta^{*}\right)$ and $\Psi_{\text {calc }}^{*}\left(\Theta^{*}\right)$, on the basis of which a command is formed to turn off the phase current $I_{\mathrm{ph}}^{*}\left(\Theta^{*}\right)$ takes place in the vicinity of the agreed position of the teeth (close to the boundary of the transition of the SRM from motor mode to the generator operating mode [10]).

However, with a slight decrease in speed to $\omega^{*}=0,58$, the rate of increase in current, as well as $\Psi_{\text {com }}^{*}\left(\Theta^{*}\right)$ and $\Psi_{\text {calc }}^{*}\left(\Theta^{*}\right)$ increase, but to a different extent. In this case, the nature of the change in the curves of $\Psi_{\text {com }}^{*}\left(\Theta^{*}\right)$ and $\Psi_{\text {calc }}^{*}\left(\Theta^{*}\right)$ (dashed lines) becomes such that the condition for disconnecting the phase is not fulfilled (there is no intersection point of these dependencies). As a result, the phase shutdown command is not received. The SRM goes into the zone of the generator mode with a positive voltage on phase and, as a result, there is a sharp increase in the current $I_{\mathrm{ph}}^{*}\left(\Theta^{*}\right)$ in it as shown in dashed line in Figure 10 (a). The simulation showed that at $K_{E}>1$, as the speed decreases in the stable switching mode, the ratio of the SRD parameters becomes such that the $\Psi_{\text {calc }}^{*}\left(\Theta^{*}\right)$ and $\Psi_{\text {com }}^{*}\left(\Theta^{*}\right)$ curves for each phase are deformed towards their approach. A situation arises in which even a slight external disturbance leads to a premature intersection of the $\Psi_{\text {calc }}^{*}\left(\Theta^{*}\right)$ and $\Psi_{\text {com }}^{*}\left(\Theta^{*}\right)$ curves and, as a result, to false phase switching of the SRM, i.e., to emergency mode SRD. As an example, by analogy with the previous case, we consider the operation of the SRD on the border of the emergency mode at $K_{E}=1,4$ as shown in zone I in Figure 11. At the speed $\omega^{*}=4$, as can be seen from Figure 15, the $\Psi_{\text {calc }}^{*}\left(\Theta^{*}\right)$ and $\Psi_{\text {com }}^{*}\left(\Theta^{*}\right)$ curves are closest to each other at the beginning of the phase switching cycle (in the vicinity of the point "a" - the breakpoint of the $\Psi_{\text {com }}^{*}\left(\Theta^{*}\right)$ curve at the level $\Psi_{\text {min }}^{*}$ ).

Investigation of faulty behavior of the sensorless control switched reluctance ... (Alexander Krasovsky) 
Nevertheless, we have the mode of stable switching of the SRM with the corresponding law of the change in phase currents. This mode corresponds to the traditional alternation of phase switching of a four-phase SRM $-1,2,3,4,1, \ldots$ with the same current shape $I_{\mathrm{ph}}^{*}\left(\Theta^{*}\right)$, as shown in Figure 12 in zone I.

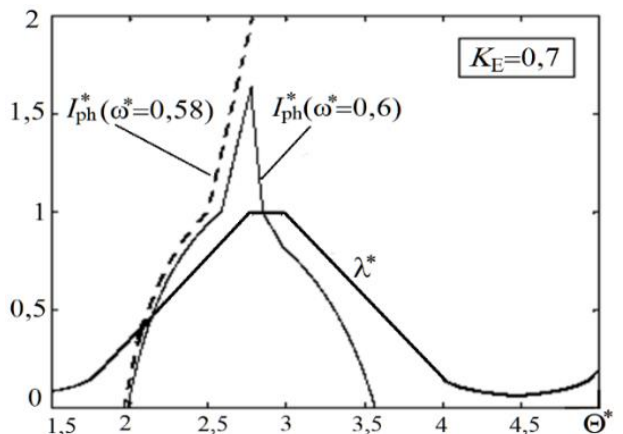

(a)

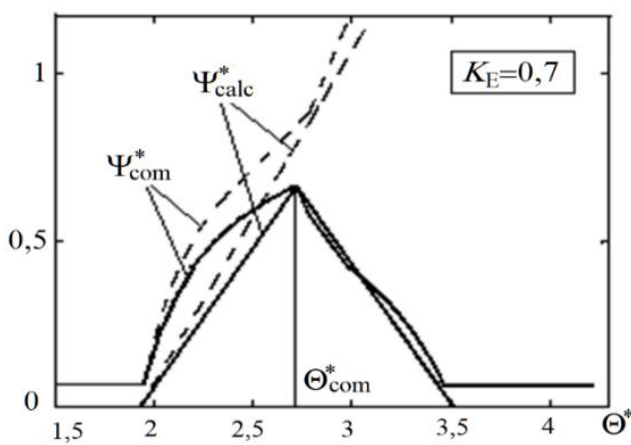

(b)

Figure 10. Illustration of the possibility switching the SRM from motor mode (a) to generator mode (b) in the low-speed zone

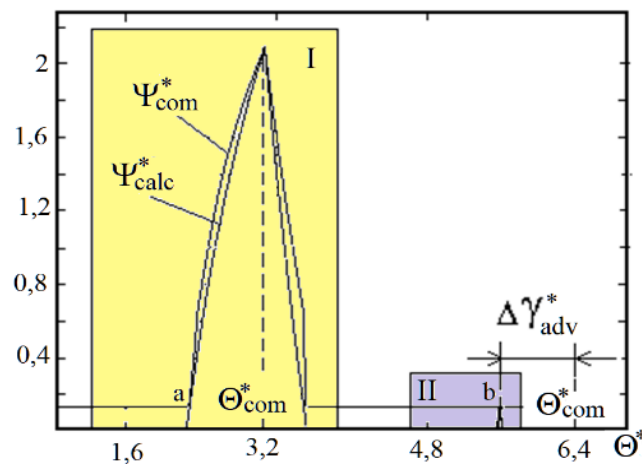

Figure 11. Explanation of the development mechanism of the anomalous SRD mode in the low-speed zone at $K_{E}=1,4$

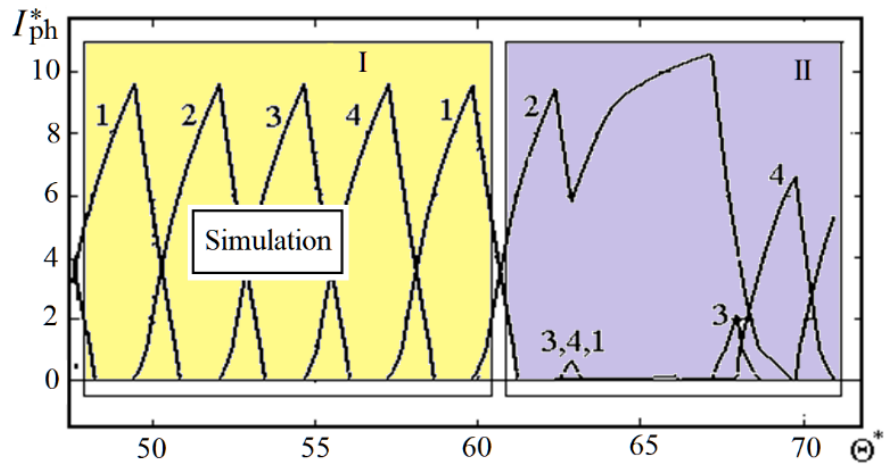

Figure 12. The calculated phase current curves at $K_{E}=1,4$

A further slight decrease in speed for any reason (for example, to $\omega^{*}=0,38$ ) leads to additional deformation of the curves $\Psi_{\text {calc }}^{*}\left(\Theta^{*}\right)$ and $\Psi_{\text {com }}^{*}\left(\Theta^{*}\right)$, and their intersection, according to which the switching moment is determined by the adopted algorithm, is shifted by the angular interval $\Delta \gamma_{\mathrm{adv}}^{*}$ to the breakpoint of the $\Psi_{\text {com }}^{*}\left(\Theta^{*}\right)$ curve (point "b" in zone II in Figure 11. This would lead to a premature switching of the currents $I_{\mathrm{ph}}^{*}\left(\Theta^{*}\right)$ of the three of the nearest phases with a high frequency. As a result, the phase during which the first false switching of the algorithm occurred ("phase 2 " in Figure 12 is switched on again before the current $I_{\mathrm{ph}}^{*}\left(\Theta^{*}\right)$ in it has time to drop to zero from the previous switching cycle.

The validity of the conclusions made regarding the causes and mechanism of the development of the anomalous SRM mode under the considered conditions can be confirmed by the experimental phase current $I_{\mathrm{ph}}(t)$ curves shown in Figure 13. As you can see, the nature of the change in the phase currents of the SRM in this figure is close to the curves of the currents $I_{\mathrm{ph}}^{*}\left(\Theta^{*}\right)$ in zone II in Figure 12 obtained by modeling. The combination of the results of modeling the behavior of the drive in the low-speed zone for $K_{E}>1$ and $K_{E}<$ 1 allows us to determine the lower boundary of stable switching of the SRM in the form of the dependence of the minimum realizable speed $\omega_{\min }$ on the value of $K_{E}$, shown in Figure 14. 


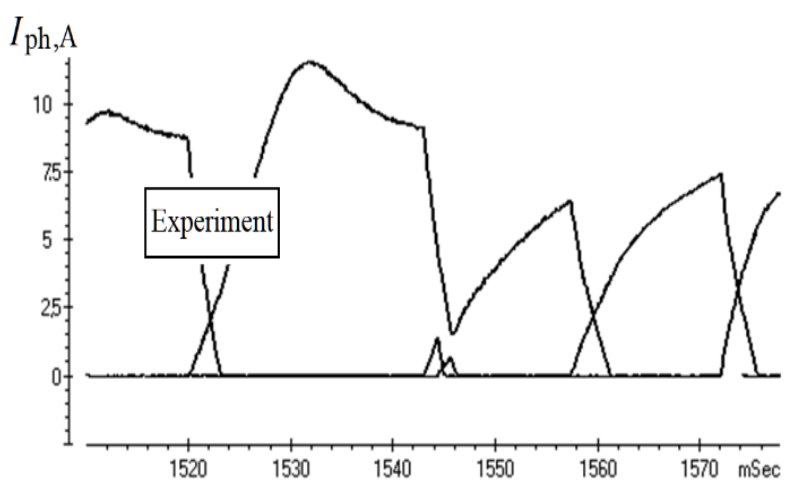

Figure 13. The experimental phase current curves at $K_{E}=1,4$

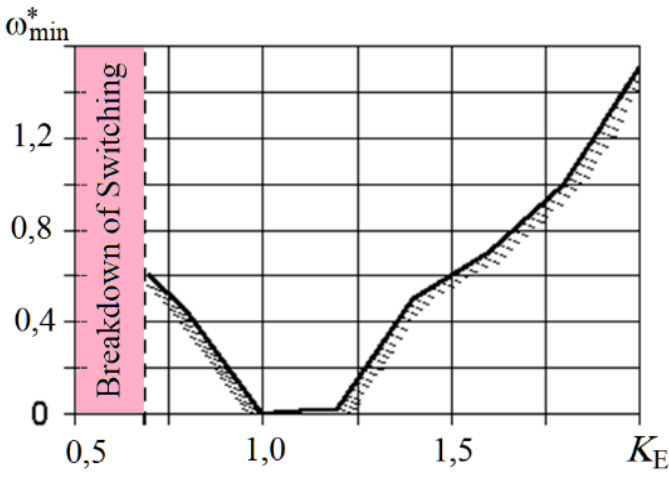

Figure 14. Lower boundary of the mode of stable switching of SRM

\section{CONCLUSION}

In the mode of sensorless control of the SRD under consideration, abnormal conditions can be induced by deviations in the values of the algorithm parameters-tuning coefficients and the shape of the switching line. With certain combinations of parameters of the sensorless control algorithm of the SRD, the cycle of its switching shifts toward the zone of creation of the braking torque in the mode of the generator with a sharp increase in the phase current. This would happen due to the accumulation of an error in the angle of switching on the phase of the SRM. The reason for the shift of the SRM switching cycle to the zone of braking torque in the regimes close to idle (no load) may be the presence of a "dead zone" introduced for stable operation of the algorithm in the field of small signals. With an increase in the load of the SRD, the main reason for the transition of the SRM to the generator mode is an unfavorable combination of scale factors in calculating the current value of phase flux linkage.

\section{REFERENCES}

[1] A.V. Isaev, at al., "Automated System for Diagnostics of Emergency Operation Modes of Electric Motor" International Conference on Industrial Engineering, Applications and Manufacturing, 2019.

[2] Weizhe Ma, Masayuki Morimoto, "Emergency drive method for EV at the inverter fault," 2010 International Conference on Electrical Machines and Systems, 2010.

[3] Georgy I. Odnokopylov, A. D. Bragin, "Algorithms of fault tolerant control of induction motor electric drive-in phase loss operate mode," 2015 International Siberian Conference on Control and Communications (SIBCON), 2015, DOI: 10.1109/SIBCON.2015.7147009.

[4] P.H. Mellor, at al., "Faulted behaviour of permanent magnet electric vehicle traction drives", IEEE International Electric Machines and Drives Conference, 2003. IEMDC'03., Madison, WI, USA, 2003, pp. 554-558, DOI: 10.1109/IEMDC.2003.1211317.

[5] Mounir Zeraoulia and Demba Diallo, "Electric Motor Drive Selection Issues for HEV Propulsion Systems: A Comparative Study," IEEE Transactions on Vehicular Technology, vol. 55, no. 6, pp. 1756-1764, 2006.

[6] Zhi Yang, at al., "Comparative study of interior permanent magnet, induction, and switched reluctance motor drives for EV," IEEE Transactions on Transportation Electrification, vol. 1, no. 3, pp. 245-254, 2015.

[7] R. Pindoriya, at al., "Comparative analysis of permanent magnet motors and switched reluctance motors capabilities for electric and hybrid electric vehicles" IEEMA Engineer Infinite Conference (eTechNxT), pp. 1-5, 2018.

[8] Yasser Alamoudi, at al., "State-of-the Art Electrical Machines for Modern Electric Vehicles," 2019 Advances in Science and Engineering Technology International Conferences (ASET), 2019, pp. 1-8.

[9] Miller T.J.E., "Electronic control of switched reluctance machines", Oxford: Newnes, pp. 272, 2001.

[10] Krishnan R., "Switched reluctance motor drives: modeling, simulation, analysis, design, and applications," Cambridge: CRC Press, pp. 432, 2001.

[11] Berker Bilgin, at al., "Switched Reluctance Motor Drives, Fundamentals to Application," CRC Press, pp. 824, 2018.

[12] Alain Glumineau, Jesús de León Morales, "Sensorless AC Electric Motor Control," Springer Internation publishing, pp. 244, 2015.

[13] Ganapathy Ram A, Santha K R., "Review of Sliding Mode Observers for Sensorless Control of Permanent Magnet Synchronous Motor Drives," International Journal of Power Electronics and Drive System (IJPEDS), vol. 9, no. 1, pp. 46-54, 2018.

[14] J. Zhang, at al., "A Simple Flux Model Based Observer for Sensorless Control of Switched Reluctance Motor," APEC 07 -Twenty-Second Annual IEEE Applied Power Electronics Conference and Exposition, pp. 587-592. 
[15] J. Cai, Z. Deng, "A Position Sensorless Control of Switched Reluctance Motors Based on Phase Inductance Slope," Journal of Power Electronics, vol. 13, no. 2, pp. 264-274, 2013.

[16] M. Ehsani, B. Fahimi, "Elimination of position sensors in switched reluctance motor drives: state of the art and future trends," IEEE Transactions on Industrial Electronics, vol. 49, no. 1, pp. 40-47, 2002.

[17] G. Lopez, P. C. Kjaer, and T. J. E. Miller, "High-grade position estimation for SRM drives using flux linkage/current correction model," in Conf. Rec. IEEE-IAS Annu. Meeting, vol. 1, pp. 731-738, 1998.

[18] J. Cai, Z. Deng, "A Position Sensorless Control of Switched Reluctance Motors Based on Phase Inductance Slope", Journal of Power Electronics, vol. 13, no. 2, pp. 264-274, 2013.

[19] Krasovsky A., at al., "Simulation of the Sensorless Switched Reluctance Motor Drives" IEEE 60th Scientific Conference on Power and Electrical Engineering of Riga Technical University (RTUCON), 2019.

[20] Haitham Abu-Rub, at al., "High Perfomance Control of AC Drives with MATLAB/SIMULINK Models," John Wiley \& Sons Ltd, pp. 492, 2012.

[21] Harold Klee, at al., "Simulation of Dynamic Systems with MATLAB and Simulink," CRC Press, pp. 832, 2018.

[22] R.S. Preethishri, J. Anitha Roseline "Switched Reluctance Motor (SRM) Development by using matlab-simulink" 2nd International Conference on Power and Embedded Drive Control (ICPEDC), 2019.

[23] Dimitar Kirov Yankov, Tsvetana Grigorova Grigorova, Emil Ivanov Dinkov, "Modeling of a tree-phase 12/8 pole switched reluctance motor in MATLAB," 2019 IEEE XXVIII International Scientific Conference Electronics (ET), DOI: 10.1109/ET.2019.8878654.

[24] Krasovsky A., "Simulation and analysis of improved direct torque control of switched reluctance machine," Indonesian Journal of Electrical Engineering and Computer Science, vol. 18, no. 1, pp. 251-260, 2019.

[25] Ehab Sayed, at al., "A MATLAB Toolbox for Adjoint-Based Sensitivity Analysis of Switched Reluctance Motors," 2018 IEEE Electrical Power and Energy Conference (EPEC), 2018. 